\title{
A randomized phase III trial of adjuvant chemotherapy with irinotecan, leucovorin and fluorouracil versus leucovorin and fluorouracil for stage II and III colon cancer: A Hellenic Cooperative Oncology Group study
}

Christos A Papadimitriou ${ }^{1 *}$, Pavlos Papakostas², Maria Karina ${ }^{3}$, Lia Malettou', Meletios A Dimopoulos?', George Pentheroudakis ${ }^{5}$, Epaminontas Samantas ${ }^{6}$, Aristotelis Bamias ${ }^{1}$, Dimosthenis Miliaras ${ }^{7}$, George Basdanis $^{8}$, Nikolaos Xiros ${ }^{9}$, George Klouvas ${ }^{10}$, Dimitrios Bafaloukos ${ }^{11}$, Georgia Kafiri ${ }^{12}$, Irene Papaspirou ${ }^{13}$, Dimitrios Pectasides ${ }^{9}$, Charisios Karanikiotis $^{14}$, Theofanis Economopoulos ${ }^{9}$, Ioannis Efstratiou ${ }^{15}$, Ippokratis Korantzis ${ }^{3}$, Nikolaos Pisanidis ${ }^{16}$, Thomas Makatsoris $^{17}$, Fotini Matsiakou ${ }^{2}$, Gerasimos Aravantinos ${ }^{6}$, Haralabos P Kalofonos ${ }^{17}$, George Fountzilas ${ }^{3}$

\begin{abstract}
Background: Colon cancer is a public health problem worldwide. Adjuvant chemotherapy after surgical resection for stage III colon cancer has been shown to improve both progression-free and overall survival, and is currently recommended as standard therapy. However, its value for patients with stage II disease remains controversial. When this study was designed 5-fluorouracil (5FU) plus leucovorin (LV) was standard adjuvant treatment for colon cancer. Irinotecan (CPT-11) is a topoisomerase I inhibitor with activity in metastatic disease. In this multicenter adjuvant phase III trial, we evaluated the addition of irinotecan to weekly 5FU plus LV in patients with stage II or III colon cancer.
\end{abstract}

Methods: The study included 873 eligible patients. The treatment consisted of weekly administration of irinotecan $80 \mathrm{mg} / \mathrm{m}^{2}$ intravenously (IV), LV $200 \mathrm{mg} / \mathrm{m}^{2}$ and $5 F U 450 \mathrm{mg} / \mathrm{m}^{2}$ bolus (Arm A) versus LV $200 \mathrm{mg} / \mathrm{m}^{2}$ and $5 F U 500$ $\mathrm{mg} / \mathrm{m}^{2}$ IV bolus (Arm B). In Arm A, treatments were administered weekly for four consecutive weeks, followed by a two-week rest, for a total of six cycles, while in Arm B treatments were administered weekly for six consecutive weeks, followed by a two-week rest, for a total of four cycles. The primary end-point was disease-free survival (DFS) at three years.

Results: The probability of overall survival (OS) at three years was 0.88 for patients in Arm A and 0.86 for those in Arm B, while the five-year OS probability was 0.78 and 0.76 for patients in Arm A and Arm B, respectively $(P=$ 0.436). Furthermore, the probability of DFS at three years was 0.78 and 0.76 for patients in Arm A and Arm B, respectively $(P=0.334)$. With the exception of leucopenia and neutropenia, which were higher in patients in Arm A, there were no significant differences in Grades 3 and 4 toxicities between the two regimens. The most frequently recorded Grade 3/4 toxicity was diarrhea in both treatment arms.

Conclusions: Irinotecan added to weekly bolus 5FU plus LV did not result in improvement in disease-free or overall survival in stage II or III colon cancer, but did increase toxicity.

Trial registration: Australian New Zealand Clinical Trials Registry: ACTRN12610000148077

\footnotetext{
* Correspondence: chr_papadim@yahoo.gr

'Department of Clinical Therapeutics, "Alexandra" Hospital, University of

Athens School of Medicine, Athens, Greece

Full list of author information is available at the end of the article
} 


\section{Background}

Colorectal cancer is one of the world's most common malignancies. After lung cancer, it is the most frequent cause of cancer-related mortality in the Western World [1]. Despite curative surgery in those presenting early, the risk of recurrence is significantly high with a mortality rate still close to $40 \%$. Hence, much work has been done in search of effective adjuvant therapy for the eradication of disseminated micrometastases. In colon cancer, chemotherapy is the principal adjuvant therapy and the addition of radiotherapy to chemotherapy has not been shown to improve outcome [2]. In the absence of any further treatment after resection of the primary tumor, five-year survival rates are principally determined by the stage of the tumor at the time of resection. Studies performed in the late 1980s demonstrated that adjuvant fluorouracil (5FU) plus levamisole improved survival in patients with a resected stage III colon cancer. Further studies performed in the mid-1990s established 5FU plus leucovorin (LV) administered for approximately six months as a standard postoperative treatment. The therapeutic potential of systemic treatments for colorectal cancer has expanded rapidly during the past 10 years, with the introduction of oral fluoropyrimidines, oxaliplatin, and irinotecan [3-6].

Irinotecan is a topoisomerase I inhibitor with activity in metastatic colorectal cancer, alone or in combination with other agents $[7,8]$, in both first- and secondline treatment of metastatic disease [9-13]. Cunningham et al. [14] randomized, in a 2:1 ratio, patients with metastatic colorectal cancer, which had progressed within six months of treatment with $5 \mathrm{FU}$ to receive either irinotecan with supportive care or supportive care alone and demonstrated a one-year survival rate 2.5 times greater for the irinotecan group than that achieved with best supportive care alone. Two further randomized trials of first-line therapy using irinotecan with or without 5FU plus LV demonstrated statistically significant survival advantages for the combination regimen $[15,16]$. As a result, the weekly irinotecan plus $5 \mathrm{FU}$ plus $\mathrm{LV}$ regimen became standard for first-line metastatic colorectal cancer. The improvements in response rate, progression-free survival (PFS), and overall survival (OS) achieved with the incorporation of irinotecan in the systemic treatment of patients with metastatic colorectal cancer encouraged its testing in the adjuvant setting, especially in patients with stage III disease. We performed a randomized controlled trial of the combination of weekly irinotecan plus LV plus 5FU versus a standard weekly schedule 5FU plus LV in the adjuvant setting following curative resection of stage II and III colon cancer.

\section{Methods}

\section{Patient selection}

All eligible patients had histologically confirmed colon adenocarcinoma and underwent complete surgery for stages B2 and C disease with neither gross nor microscopic evidence of residual tumor. The patients entered the study within three to six weeks after surgery; had not received any prior chemotherapy; were aged at least 18 years; had a World Health Organization (WHO) performance status $\leq 2$; and should have no history of other malignancies except of adequately treated carcinoma in situ of the uterine cervix, or curatively treated non-melanomatous skin cancer or serious illness that would preclude protocol chemotherapy. Other laboratory eligibility requirements included absolute neutrophil count $\geq 1,500 / \mu \mathrm{l}$, platelet count $\geq 100,000 / \mu \mathrm{l}$, serum creatinine $<1.5 \mathrm{mg} / \mathrm{dl}$, and alanine transaminase (ALT) and aspartate aminotransferase (AST) levels less than twice the institutional upper limit of normal. Non eligibility criteria were histology other than adenocarcinoma, incomplete resection, myocardial infarction within the last six months or uncontrolled coronary insufficiency, inflammatory intestinal disease, and pregnant or nursing women. The clinical protocol and collateral translational studies were approved by the Hellenic Cooperative Oncology Group (HeCOG) Protocol Review Committee. The study was conducted in accordance with the ethical principles of the Declaration of Helsinki and Good Clinical Practice. Our study was also registered at the Australian New Zealand Clinical Trials Registry (ACTRN12610000148077). Before randomization all patients provided written informed consent and eligibility was confirmed by a protocol-specific checklist.

\section{Evaluation}

Before study entry, all patients underwent a complete physical examination, assessment of performance status, complete blood cell (CBC) count and differential, liver and kidney function tests, serum carcinoembryonic antigen (CEA) and cancer antigen (CA) 19-9, urinalysis, electrocardiogram (ECG), pelvic and abdominal computed tomography, and chest X-ray with computed tomography of the chest when clinically indicated. During treatment, clinical evaluation and $\mathrm{CBC}$ were performed every week. Furthermore, biochemistry laboratory evaluations were performed every two weeks until the end of the treatment, and then every three months thereafter. Abdominal and pelvic CT scans and chest-X-ray were performed at the end of the study, and then every six months thereafter. However, CT scans were repeated earlier whenever clinically indicated depending on the discretion of the investigator. Endoscopy was performed annually $[12,17]$. 


\section{Treatment}

Eligible and registered patients were randomly assigned using central registration to receive either irinotecan plus LV plus 5FU (Arm A) or LV plus 5FU (Arm B). Chemotherapy in Arm A consisted of irinotecan $80 \mathrm{mg} /$ $\mathrm{m}^{2}$ in $250 \mathrm{ml}$ normal saline, as a 90-minute intravenous (IV) infusion, followed by LV $200 \mathrm{mg} / \mathrm{m}^{2}$ in $500 \mathrm{ml}$ normal saline, IV over two hours, and 5FU $450 \mathrm{mg} / \mathrm{m}^{2}$, as IV rapid administration, 60 minutes after initiation of LV infusion. Treatments were administered weekly for four consecutive weeks, followed by a two-week rest, for a total of six cycles. The LV plus 5FU group (Arm B) received chemotherapy consisting of $\mathrm{LV} 200 \mathrm{mg} / \mathrm{m}^{2}$ by IV injection over two hours, with a bolus of $5 \mathrm{FU}$ $500 \mathrm{mg} / \mathrm{m}^{2}$ administered by IV injection at 60 minutes after initiation of LV. Treatments were administered weekly for six consecutive weeks, followed by a twoweek rest, for a total of four cycles.

\section{Dose modification}

Toxicities were graded using the WHO common criteria. Dose adjustments of the drugs or treatment delays were decided according to the worst toxicity grade at preceding cycle. The dose modification was determined according to the body system showing the greatest toxicity. Chemotherapy was interrupted if Grade more than 2 toxicity was encountered and was restarted when toxicity had resolved to $\leq$ Grade 1 . In case of diarrhea, the patients underwent supportive care and also intensive treatment with loperamide and were hospitalized if necessary. The doses of irinotecan and 5FU were reduced by $20 \%$ or $30 \%$ in the case of Grades 2 and 3 diarrhea, respectively. In the presence of Grades 1, 2 and 3 hematological toxicity, treatment was delayed for at least one week until hematological recovery without dose reductions in further infusions, while in the case of Grade 4 hematological toxicity, irinotecan as well as the 5 FU dose was reduced by $20 \%$ in all the subsequent courses.

There was no re-escalation for patients experiencing bone marrow or gastrointestinal toxicity requiring dose modification. In the case of hand-foot syndrome (Grade 3 or 4 ), only the dose of $5 F U$ was to be reduced by $20 \%$. In the case of angina or myocardial infarction the treatment would be ceased. Furthermore, for Grade 3 or 4 mucositis, there was a possibility for restarting the treatment after one week delay, if toxicity had resolved to $\leq$ Grade 1 and the dose of irinotecan and $5 \mathrm{FU}$ was to be reduced by $20 \%$. In the presence of any Grade 4 toxicity, except for gastrointestinal or hematological toxicity, the patients were withdrawn from the study. No prophylactic treatment was permitted for diarrhea. Specific guidelines for curative treatment of delayed diarrhea were provided, which recommended loperamide $2 \mathrm{mg}$ every $2 \mathrm{~h}$ for $12 \mathrm{~h}$ after the last loose stool, for a maximum of 48 consecutive hours. If diarrhea was not controlled after $48 \mathrm{~h}$ of non-stop loperamide intake, or if the patient was dehydrated, loperamide was stopped and the patient was hospitalized for intravenous fluids.

\section{Statistical analysis}

For a two-sided test at the 5\% level of significance and a power of $80 \%$, the number of patients required to detect a difference between the two treatment arms within $5 \%$ $( \pm 2.5 \%)$ to the baseline rate of $80 \%$ in DFS at the threeyear time point [18] was 870 patients. Taking into account a $3 \%$ withdrawal, 900 patients (450 per group) were needed to enter the study with an accrual rate of 170 patients per year and a corresponding maximum study duration of 9.9 years. An interim analysis based on the O'Brien Fleming boundary values was performed when half $(50 \%)$ of the endpoints (161 relapses) had been reached. The study would be ended prematurely if either a significant difference was detected or the alternative was rejected at the interim analysis. No significant difference in DFS rate was detected $(P=0.112)$ and the study was continued to completion.

Overall survival (OS) was measured from the date of randomization until death from any cause. Surviving patients were censored at the date of last contact. DFS was measured from the date of randomization until recurrence of tumor or secondary malignancy or death from disease without relapse. "Secondary malignancy" includes any cancer and not just another colorectal cancer. Death from disease without relapse means death without documented progression of the disease, which includes deaths from any cause.

Continuous variables were presented as medians with the corresponding range and categorical variables as frequencies with the respective percentages. The Fisher's exact test and the non-parametric Mann-Whitney were used for comparing patient and tumor characteristics. Time to event distributions were estimated using Kaplan-Meier curves and compared using the log-rank test. The Cox proportional hazards models were used to assess the relationship of OS and DFS with various clinical and histological variables. The backward selection procedure with removal criterion $P>0.10$ based on Likelihood ratio test was performed to identify significant variables among the following: treatment group (Arm A versus Arm B), age, histology grade (I versus II to III), Dukes stage (B versus $\mathrm{C}$ ), number of examined lymph nodes, number of involved lymph nodes, obstruction (no versus yes), perforation (no versus yes), primary site (right colon versus left colon versus sigmoid). All statistical tests were two-sided and performed at a significance level of 0.05. The SPSS software was used for statistical analysis (SPSS for Windows, version 18.0 PASW 
Statistics, IBM, Chicago, IL). Analysis was carried out following the modified intention-to-treat principle, that is, including all eligible patients.

\section{Results}

Between January 1999 and September 2004, 909 patients were randomly assigned to irinotecan plus 5FU plus LV or to $5 \mathrm{FU}$ plus LV. Thirty-six patients (4\%) were ineligible: 15 had metastatic disease, 11 had rectal cancer, 5 had non radical surgery, 3 had a history of other malignancy, 1 had positive margins, and 1 had ovarian adenocarcinoma. The outline of the study is shown in Figure 1. For 14 patients medical records were incomplete at the time of the analysis ( 8 from Arm A and 6 from Arm B). Furthermore, six patients in Arm A and eight patients in Arm B never started on chemotherapy; these patients were included in the efficacy analysis according to the intent-to-treat method, but were excluded from the toxicity and treatment characteristics analyses. Four patients randomized to Arm A received Arm B treatment and vice versa. Patient characteristics were well balanced for age, gender, $\mathrm{T}$ and $\mathrm{N}$ stage, median number of nodes reported, and the presence of perforation or obstruction (Table 1).

Out of the 873 eligible patients, 148 discontinued treatment, 77 (18\%) patients in Arm A and 71 (17\%) in

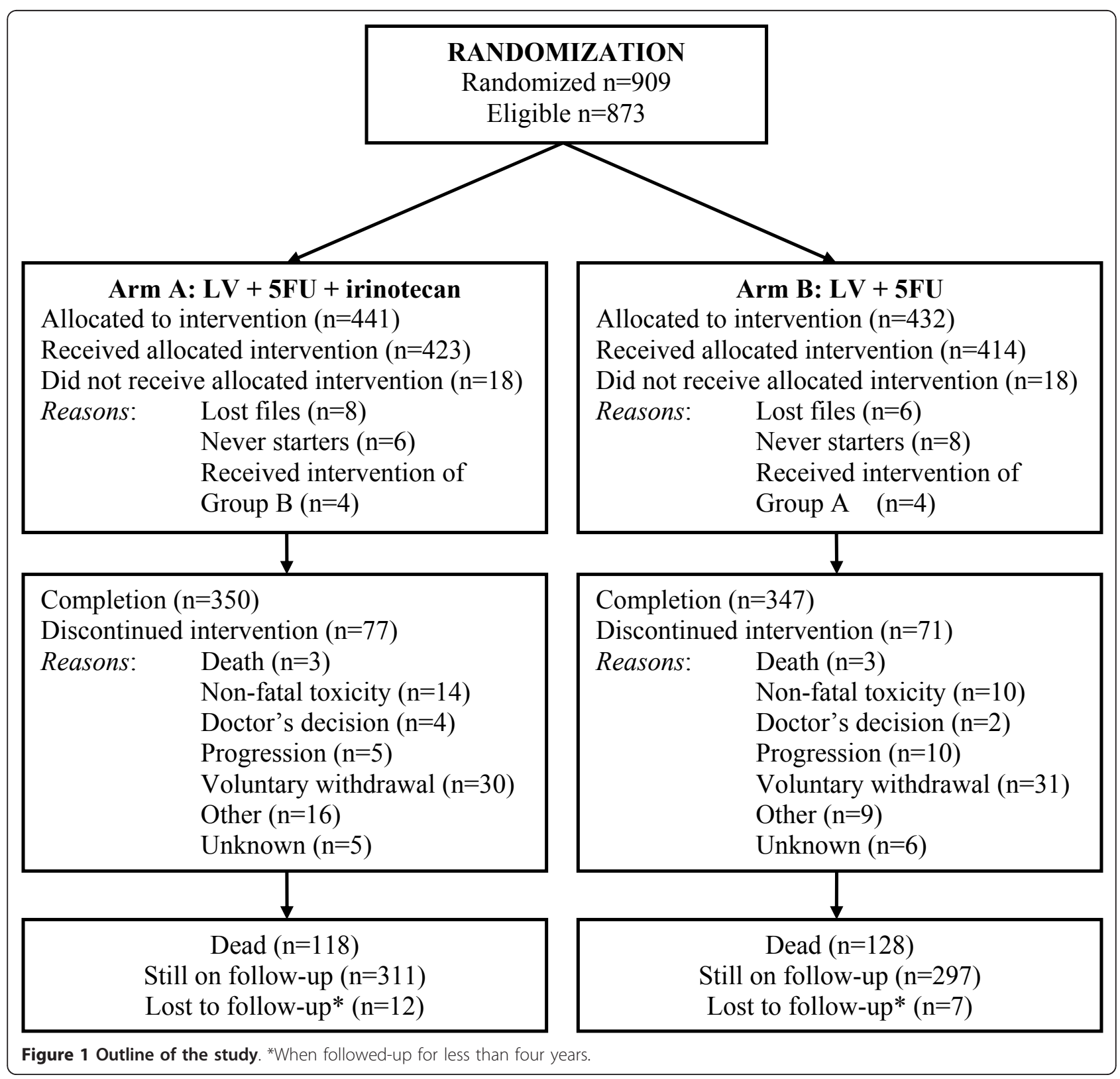


Table 1 Patient characteristics

\begin{tabular}{|c|c|c|c|}
\hline Characteristic & $\begin{array}{c}\text { Arm A: IRI+LV+5FU } \\
N=441(\%)\end{array}$ & $\begin{array}{c}\text { Arm B: LV+5FU } \\
N=432(\%)\end{array}$ & $P$ \\
\hline Age (years) & & & 0.336 \\
\hline Median & 65 & 65 & \\
\hline Range & 26 to 79 & 24 to 79 & \\
\hline Sex & & & 0.892 \\
\hline Female & $206(47)$ & $199(46)$ & \\
\hline Male & $235(53)$ & $233(54)$ & \\
\hline Stage (Dukes) & & & 0.946 \\
\hline B & $214(48)$ & $211(49)$ & \\
\hline C & $227(52)$ & $221(51)$ & \\
\hline Primary tumor $(\mathrm{T})$ & & & 0.891 \\
\hline $\mathrm{T} 1$ and $\mathrm{T} 2$ & $29(6)$ & $27(6)$ & \\
\hline $\mathrm{T} 3$ and $\mathrm{T} 4$ & $405(92)$ & $399(93)$ & \\
\hline Unknown & $7(2)$ & $6(1)$ & \\
\hline $\begin{array}{l}\text { Regional lymph } \\
\text { nodes }(\mathrm{N})\end{array}$ & & & 0.350 \\
\hline No & $210(48)$ & $208(48)$ & \\
\hline N1 & $153(35)$ & $134(31)$ & \\
\hline N2 & $70(16)$ & $82(19)$ & \\
\hline Unknown & $8(2)$ & $8(2)$ & \\
\hline Histology grade & & & 0.048 \\
\hline । & $40(9)$ & $47(11)$ & \\
\hline$\|$ & $334(76)$ & $296(68)$ & \\
\hline III & $62(14)$ & $84(20)$ & \\
\hline Unknown & $5(1)$ & $5(1)$ & \\
\hline $\begin{array}{l}\text { Number of lymph } \\
\text { nodes examined }\end{array}$ & & & 0.354 \\
\hline Median & 14 & 14 & \\
\hline Range & 0 to 96 & 0 to 70 & \\
\hline $\begin{array}{l}\text { Number of lymph } \\
\text { nodes involved* }\end{array}$ & & & 0.271 \\
\hline Median & 2 & 3 & \\
\hline Range & 1 to 23 & 1 to 27 & \\
\hline Site of disease & & & 0.640 \\
\hline Cecum & $88(20)$ & $95(22)$ & \\
\hline Ascending colon & $67(15)$ & $67(16)$ & \\
\hline Transverse colon & $29(7)$ & $30(7)$ & \\
\hline Descending colon & $36(8)$ & $44(10)$ & \\
\hline Sigmoid & $218(49)$ & $194(45)$ & \\
\hline Unknown & $4(1)$ & $2(0.5)$ & \\
\hline Perforation & & & 0.897 \\
\hline Yes & $406(92)$ & $398(92)$ & \\
\hline No & $33(7)$ & $31(7)$ & \\
\hline Unknown & $2(1)$ & $3(1)$ & \\
\hline Obstruction & & & 0.277 \\
\hline Yes & $386(88)$ & $365(84)$ & \\
\hline No & $54(12)$ & $64(15)$ & \\
\hline Unknown & $1(0.2)$ & $3(1)$ & \\
\hline
\end{tabular}

*Only for stage $C$

disease.
Arm B, respectively. The most common reason for treatment discontinuation was voluntary withdrawal (30 patients in Arm A and 31 patients in Arm B). Additional reasons for treatment discontinuation were toxicity (14 patients in Arm A and 10 patients in Arm B), disease progression (5 versus 10$)$, death ( 3 versus 3 ), physician's decision (4 versus 2 ) and others (16 versus 9). In all, 350 (79\%) patients randomized in Arm A and 347 (80\%) in Arm B completed treatment.

\section{Efficacy}

The median follow-up time was 77.4 months (range, 0.1 to 124.7). There were no significant differences between irinotecan plus LV plus 5FU and LV plus $5 \mathrm{FU}$ in OS and DFS (Table 2). Survival curves are presented in Figures 2 and 3 . The probability of OS at three years was 0.88 for patients in Arm A and 0.86 for those in Arm B, while the five-year OS probability was 0.78 and 0.76 for patients in Arm A and Arm B, respectively. Furthermore, the probability of DFS at three years was 0.78 and 0.76 for patients in Arm A and Arm B, respectively. Five-year DFS probability was 0.70 for Arm A and 0.68 for Arm B.

When patients were analyzed by stage (Table 3 ), those with Dukes B disease had an OS probability at three years of 0.93 in Arm A and 0.89 in Arm B, while their five-year OS probability was 0.86 and 0.83 in Arms A and B, respectively. Furthermore, the probability of DFS at three years was 0.86 and 0.83 for patients in Arms A and $\mathrm{B}$, respectively. The three-year OS probability for patients with stage $C$ disease was 0.79 in Arm A and 0.82 for those in Arm B, while their five-year OS

Table 2 Disease-free survival and overall survival

\begin{tabular}{lccc}
\hline & $\begin{array}{c}\text { Arm A IRI+LV } \\
+5 F U\end{array}$ & $\begin{array}{c}\text { Arm B LV } \\
+5 F U\end{array}$ & $\begin{array}{c}P \text { (log- } \\
\text { rank) }\end{array}$ \\
\hline $\begin{array}{l}\text { Disease-free survival } \\
\text { (DFS) }\end{array}$ & & & 0.436 \\
$\quad$ Progressions, n (\%) & $110(24.9)$ & $112(25.9)$ & \\
Median (months) & Not reached yet & Not reached & \\
& & yet & \\
three-year DFS (\%) & 78 & 76 & \\
five-year DFS (\%) & 70 & 68 & \\
seven-year DFS (\%) & 66 & 63 & \\
Overall survival (OS) & & & \\
Deaths, n (\%) & $118(26.8)$ & $128(29.6)$ & \\
Median (months) & Not reached yet & Not reached & \\
& & yet & \\
three-year OS (\%) & 88 & 86 & \\
five-year OS (\%) & 78 & 76 & \\
seven-year OS (\%) & 72 & 69 & \\
\hline
\end{tabular}




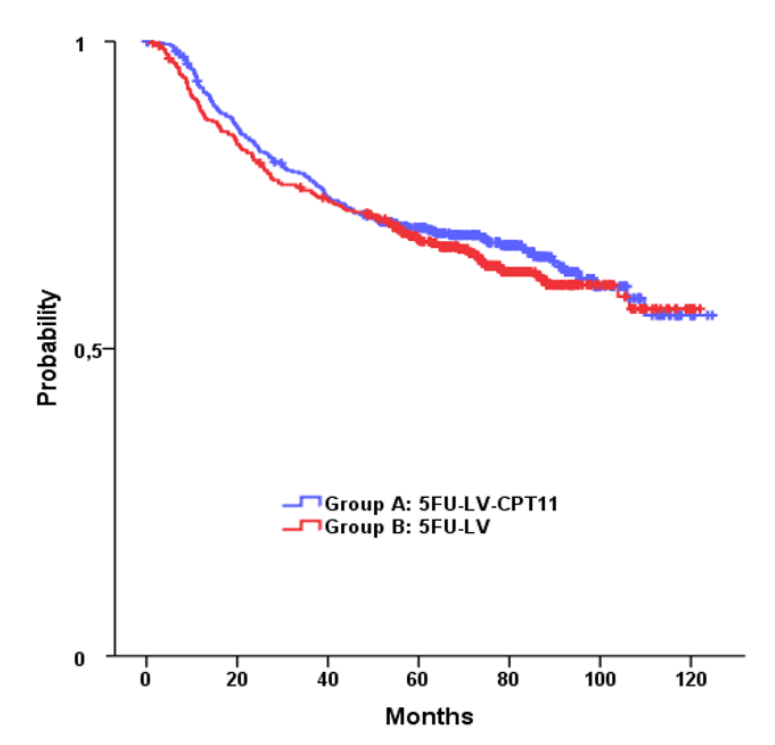

Figure 2 Kaplan-Meier curves for the DFS in the two groups

probability was 0.71 and 0.68 in Arms A and B, respectively. DFS probability at three years was 0.69 and 0.68 for patients in Arms A and B, respectively. Only 24 (2.7\%) patients out of 873 who were analyzed developed secondary malignancy (10 in arm A and 14 in arm B). Our results do not change if we use the definition of DFS as used in the MOSAIC trial (that is, not including secondary malignancies) [19].

The median relative dose intensity for $5 \mathrm{FU}$ was 0.97 (range 0.24 to 1.27 ) in Arm A and 0.86 (0.14 to 1.10) in Arm B $(P<0.001)$. The relative dose intensity for irinotecan in Arm A was 0.95 (range 0.21 to 1.27 ) (Table 4).

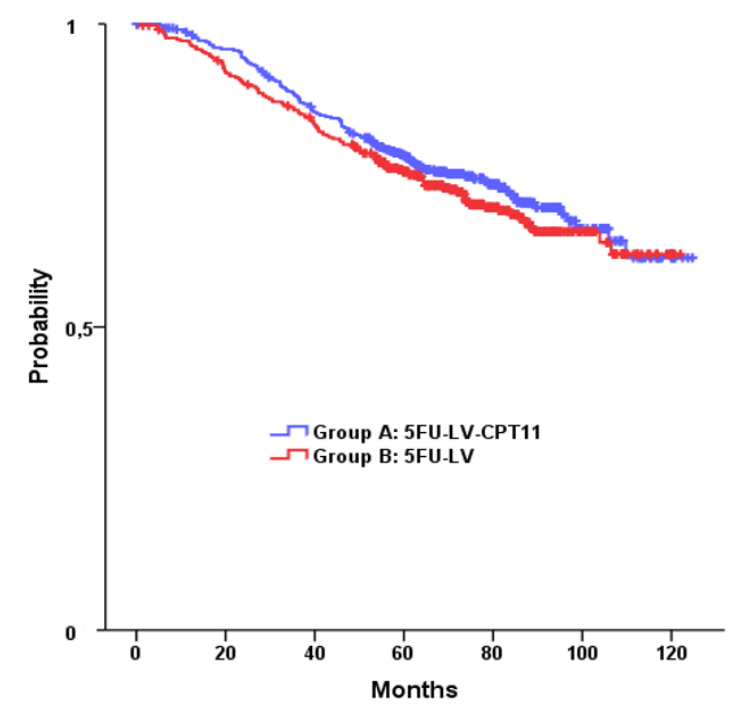

Figure 3 Kaplan-Meier curves for the OS in the two groups.
Table 3 Disease-free survival and overall survival stratified by stage

\begin{tabular}{|c|c|c|c|}
\hline & $\begin{array}{c}\text { Arm A IRI+LV } \\
+5 \mathrm{FU}\end{array}$ & $\begin{array}{l}\text { Arm B LV } \\
+5 \mathrm{FU}\end{array}$ & $P$ (log-rank) \\
\hline \multicolumn{4}{|l|}{ Stage B $(n=425)$} \\
\hline $\begin{array}{l}\text { Disease-free survival } \\
\text { (DFS) }\end{array}$ & & & 0.561 \\
\hline Progressions, $n$ (\%) & $36(16.8)$ & $35(16.6)$ & \\
\hline Median (months) & Not reached yet & $\begin{array}{c}\text { Not reached } \\
\text { yet }\end{array}$ & \\
\hline three-year DFS (\%) & 86 & 83 & \\
\hline five-year DFS (\%) & 79 & 78 & \\
\hline seven-year DFS (\%) & 75 & 73 & \\
\hline Overall survival (OS) & & & 0.389 \\
\hline Deaths, $n$ (\%) & $39(18.2)$ & $46(21.8)$ & \\
\hline Median (months) & Not reached yet & $\begin{array}{c}\text { Not reached } \\
\text { yet }\end{array}$ & \\
\hline three-year OS (\%) & 93 & 89 & \\
\hline five-year OS (\%) & 86 & 83 & \\
\hline seven-year OS (\%) & 80 & 78 & \\
\hline \multicolumn{4}{|l|}{ Stage $C(n=448)$} \\
\hline $\begin{array}{l}\text { Disease-free survival } \\
\text { (DFS) }\end{array}$ & & & 0.515 \\
\hline Progressions, $n$ (\%) & $74(32.6)$ & 77 (34.8) & \\
\hline Median (months) & 106 & 104 & \\
\hline three-year DFS (\%) & 69 & 68 & \\
\hline five-year DFS (\%) & 60 & 57 & \\
\hline seven-year DFS (\%) & 57 & 52 & \\
\hline Overall survival (OS) & & & 0.539 \\
\hline Deaths, $n(\%)$ & 79 (34.8) & $82(37.1)$ & \\
\hline Median (months) & Not reached yet & $\begin{array}{c}\text { Not reached } \\
\text { yet }\end{array}$ & \\
\hline three-year OS (\%) & 83 & 83 & \\
\hline five-year OS (\%) & 71 & 68 & \\
\hline seven-year OS (\%) & 64 & 59 & \\
\hline
\end{tabular}

In univariate analysis, the most important prognostic factors were age at diagnosis as a continuous variable hazard rate $(\mathrm{HR})=1.03,95 \% \mathrm{CI} 1.02$ to $1.05, P<0.001$ for OS and HR $=1.02,95 \% \mathrm{CI} 1.01$ to $1.03, P=0.001$ for DFS), stage C versus B (HR $=2.04,95 \%$ CI 1.57 to 2.65, $P<0.001$ for OS and HR $=2.06,95 \%$ CI 1.63 to 2.60, $P<0.001$ for DFS), T3 and T4 versus T1 and T2 disease $(\mathrm{HR}=2.72,95 \% \mathrm{CI} 1.28$ to $5.77, P=0.009$ for OS and $\mathrm{HR}=2.64,95 \%$ CI 1.36 to $5.13, P=0.004$ for DFS), number of involved lymph nodes as continuous variable $(\mathrm{HR}=1.10,95 \% \mathrm{CI} 1.08$ to $1.13, P<0.001$ for OS and HR $=1.10,95 \%$ CI 1.08 to $1.13, P<0.001$ for $\mathrm{DFS})$, the presence or not of obstruction $(\mathrm{HR}=1.35$, 95\% CI 0.97 to $1.88, P=0.076$ for OS and $\mathrm{HR}=1.37$, 95\% CI 1.01 to $1.85, P=0.041$ for DFS), and the presence or not of perforation (HR $=1.46,95 \%$ CI 0.95 to 2.52, $P=0.082$ for OS and $\mathrm{HR}=1.61,95 \%$ CI 1.10 to $2.35, P=0.013$ for DFS). 
Table 4 Dose intensity (DI)

\begin{tabular}{|c|c|c|c|}
\hline & $\begin{array}{c}\text { Arm A: IRI+LV+5FU } \\
N=427\end{array}$ & $\begin{array}{c}\text { Arm B: LV+5FU N } \\
=418\end{array}$ & $P$ \\
\hline \multicolumn{4}{|l|}{$\begin{array}{l}\text { DI of irinotecan } \\
\left(\mathrm{mg} / \mathrm{m}^{2} / \mathrm{wk}\right)\end{array}$} \\
\hline Median & 50.5 & $N A^{*}$ & \\
\hline Range & 11 to 68 & NA & \\
\hline \multicolumn{4}{|l|}{$\begin{array}{l}\text { Relative DI of } \\
\text { irinotecan }\end{array}$} \\
\hline Median & 0.95 & NA & \\
\hline Range & 0.21 to 1.27 & NA & \\
\hline $\begin{array}{l}\text { DI of } \mathrm{LV}\left(\mathrm{mg} / \mathrm{m}^{2} /\right. \\
\mathrm{wk})\end{array}$ & & & $\begin{array}{c}< \\
0.001\end{array}$ \\
\hline Median & 129 & 131 & \\
\hline Range & 32 to 165 & 21 to 279 & \\
\hline \multicolumn{4}{|l|}{ Relative DI of LV } \\
\hline Median & 0.96 & 0.87 & \\
\hline Range & 0.24 to 1.24 & 0.14 to 1.86 & \\
\hline $\begin{array}{l}\text { DI of } 5 \mathrm{FU} \\
\left(\mathrm{mg} / \mathrm{m}^{2} / \mathrm{wk}\right)\end{array}$ & & & $\begin{array}{c}< \\
0.001\end{array}$ \\
\hline Median & 291 & 322 & \\
\hline Range & 73 to 380 & 54 to 411 & \\
\hline \multicolumn{4}{|l|}{ Relative DI of $5 \mathrm{FU}$} \\
\hline Median & 0.97 & 0.86 & \\
\hline Range & 0.24 to 1.27 & 0.14 to 1.10 & \\
\hline
\end{tabular}

${ }^{*}$ Not applicable.

In the multivariate model, only age at diagnosis $(\mathrm{HR}=$ $1.03,95 \%$ CI 1.02 to $1.05, P<0.001)$, stage $(C$ versus $B)$ $(\mathrm{HR}=1.41,95 \% \mathrm{CI} 1.04$ to $1.92, P=0.028)$ and number of involved lymph nodes (HR $=1.09,95 \%$ CI 1.05 to $1.12, P<0.001)$ maintained predictive significance for OS. Similarly, older age $(\mathrm{HR}=1.02,95 \% \mathrm{CI} 1.01$ to $1.03, P=0.002)$, stage $(C$ versus $B)(\mathrm{HR}=1.44,95 \% \mathrm{CI}$ 1.10 to $1.90, P=0.009$ ), number of involved lymph nodes $(\mathrm{HR}=1.09,95 \% \mathrm{CI} 1.05$ to $1.12, P<0.001)$ and the presence of obstruction ( $\mathrm{HR}=1.43,95 \% \mathrm{CI} 1.05$ to $1.94, P=0.022$ ) were found to be significantly associated with poorer DFS.

\section{Toxicity}

Serious adverse events associated with each treatment regimen are listed in Table 5. Regarding toxicity, patients were analyzed according to the treatment patients actually received. With the exception of leucopenia and neutropenia, which were higher in patients in Arm A $(P=0.037$ and $P<0.001$, respectively $)$, there were no significant differences in Grades 3 and 4 toxicities between the two regimens. The most frequently recorded Grade 3/4 toxicity was diarrhea in both treatment arms, followed by neutropenia. Overall, 116 patients in group A (27\%) experienced any severe toxicity versus 76 patients in group B $(18 \%), P=0.002$. In $14(3 \%)$ patients on Arm A and $10(2 \%)$ patients on Arm B, treatment was stopped due to an adverse event.
Table 5 Incidence of Grades 3 and 4 toxicities (treatment as administered)

\begin{tabular}{lcccc}
\hline & $\begin{array}{c}\text { Arm A: IRI+LV+5FU } \\
(\mathbf{N}=\mathbf{4 2 7})\end{array}$ & $\begin{array}{c}\text { Arm B: LV+5FU } \\
(\mathbf{N}=\mathbf{4 1 8})\end{array}$ \\
\cline { 2 - 5 } & \multicolumn{2}{c}{$n$ (\%) } & \multicolumn{2}{c}{$\boldsymbol{n}(\%)$} \\
\cline { 2 - 5 } & Grade $\mathbf{3}$ & Grade 4 & Grade 3 & Grade 4 \\
\hline Leukopenia & $10(2)$ & 0 & $2(0.5)$ & 0 \\
Neutropenia & $39(9)$ & $6(1)$ & $7(2)$ & $4(1)$ \\
Anemia & $2(0.5)$ & 0 & 0 & 0 \\
Thrombocytopenia & $2(0.5)$ & 0 & 0 & 0 \\
Diarrhea & $58(14)$ & $6(1)$ & $52(12)$ & $2(0.5)$ \\
Nausea/vomiting & $7(2)$ & 0 & $1(0.2)$ & 0 \\
Alopecia & $6(1)$ & 0 & $1(0.2)$ & 0 \\
Neutropenic fever & $4(1)$ & 0 & $1(0.2)$ & 0 \\
Constipation & $2(0.5)$ & 0 & $1(0.2)$ & 0 \\
Pain & $1(0.2)$ & 0 & $2(0.5)$ & 0 \\
Hepatotoxicity & $1(0.2)$ & 0 & $2(0.5)$ & 0 \\
Mucositis & 0 & 0 & $2(0.5)$ & 0 \\
Fatigue & $1(0.2)$ & 0 & 0 & 0 \\
Watery eye & 0 & 0 & $1(0.2)$ & 0 \\
Peripheral neuropathy & 0 & 0 & $1(0.2)$ & 0 \\
Skin & 0 & 0 & $1(0.2)$ & 0 \\
\hline & & & & 0 \\
\hline
\end{tabular}

The unscheduled hospital admissions of patients due to treatment Grade 3 or 4 toxicity were 39 (9\%) in Arm A and $47(11 \%)$ in Arm B $(P=0.363)$. Granulocyte colony-stimulating factor (G-CSF) because of leucopenia/ neutropenia was administered in 135 patients, 98 (23\%) in Arm A and $37(9 \%)$ in Arm B $(P<0.001)$. Of the 246 deaths that occurred during the follow-up period, 241 (98\%) were due to colon cancer, 2 due to toxicity of the treatment (neutropenic sepsis) and 4 due to other causes such as pulmonary embolism (2 patients), acute myocardial infarction and CNS ischemia.

\section{Discussion}

This study was designed to assess the impact on the three-year disease-free survival of the addition of irinotecan to conventional adjuvant treatment with LVmodulated bolus 5FU, following curative resection of stage II or III colon cancer. Based on our experience with irinotecan's safety and efficacy [12] and on the encouraging results of several studies in patients with metastatic disease [9-14], it was anticipated that irinotecan would be an effective addition to adjuvant treatment program for colon cancer. Our study did not demonstrate a statistically significant difference in the threeyear disease-free and overall survival between the study arms.

In agreement with our study, three large prospective randomized trials evaluating the addition of irinotecan to bolus or continuous infusion of 5FU and LV had failed to show a survival benefit in colon cancer 
adjuvant setting. In the CALGB 89803 trial, Saltz et al. compared conventional bolus 5FU plus LV with or without addition of irinotecan [20]. After resection of stage III colon cancer, 1,264 patients were assigned randomly to receive a conventional regimen (weekly LV $500 \mathrm{mg} /$ $\mathrm{m}^{2}$ plus $5 \mathrm{FU} 500 \mathrm{mg} / \mathrm{m}^{2}$, administered for six consecutive weeks followed by two weeks of rest, for four cycles) or the experimental IFL regimen (weekly irinotecan $125 \mathrm{mg} / \mathrm{m}^{2}$ and LV $20 \mathrm{mg} / \mathrm{m}^{2}$ plus 5 FU $500 \mathrm{mg} / \mathrm{m}^{2}$, administered for four consecutive weeks followed by two weeks of rest, for five cycles). Lethal and nonlethal toxicity was significantly greater for IFL than for LV plus 5FU. On the other hand, no differences were found at three years for IFL compared with LV plus 5FU in the probability of overall survival, disease-free survival, or relapse-free survival; similarly, no differences were seen in five-year outcomes.

The negative results of CALGB 89803 are mirrored in two recently published European adjuvant trials of irinotecan plus continuous infusion 5FU (FOLFIRI), which failed to lengthen disease-free survival in colon cancer after surgical resection: PETACC-3 and ACCORD. More specifically, the PETACC-3 study investigated whether the addition of irinotecan to the de Gramont infusional 5FU and LV adjuvant regimen (LV5FU2) would improve DFS in patients with stage III colon cancer. The principal efficacy analysis was based on 2,094 treated patients, randomly allocated in the LV5FU2 strata. Severe gastrointestinal and hematologic toxicity was increased in patients receiving irinotecan [21]. The multicenter adjuvant phase III trial published by Ychou et al. evaluated the addition of irinotecan to LV5FU2 in colon cancer patients specifically at high risk of relapse [22]. This study randomly assigned 400 patients with either N1 tumors with obstruction/perforation or N2 tumors to LV5FU2, with or without irinotecan. Similarly, there was no evidence of improvement in DFS and OS in patients receiving irinotecan, while higher rates of Grades 3 and 4 neutropenia were observed.

Our study was designed to include both stage II and III patients in the analysis. Although historically many studies have combined the population of patients with both stage II and stage III disease, including some irinotecan adjuvant trials, the current trend is to perform separate clinical trials, since there are significant survival differences among patients with different $\mathrm{T}$ and $\mathrm{N}$ status. Some trials have been designed with a particular focus on high risk patients. Unfortunately, when high risk patients were evaluated, such as in the ACCORD study, as previously mentioned, irinotecan still did not provide significant benefit compared to $5 \mathrm{FU}$ and $\mathrm{LV}$.

In the present study, we utilized a weekly IFL regimen based on our previous experience. More specifically,
Kalofonos et al. [12] treated 55 patients with first-line chemotherapy for advanced disease with either irinotecan 80 $\mathrm{mg} / \mathrm{m}^{2}$ (7 patients) or $70 \mathrm{mg} / \mathrm{m}^{2}$ (48 patients) plus LV 200 $\mathrm{mg} / \mathrm{m}^{2}$ and $5 \mathrm{FU} 450 \mathrm{mg} / \mathrm{m}^{2}$, weekly for six weeks followed by a two-week rest period. Treatment was continued for four cycles. Because of Grades 3 and 4 diarrhea in four of the first seven patients, the study was amended to reduce the starting dose of irinotecan from 80 to $70 \mathrm{mg} / \mathrm{m}^{2}$ weekly. In another randomized phase II trial conducted by our Cooperative Group, 295 patients with metastatic colorectal cancer were randomized to receive as first-line chemotherapy either irinotecan $70 \mathrm{mg} / \mathrm{m}^{2}$ plus LV and $5 \mathrm{FU}$, as previously described, or oxaliplatin plus LV plus 5FU. Severe diarrhea occurred in $12.3 \%$ of patients of the irinotecan arm [17]. Hematological toxicity and gastrointestinal mucositis were a concern when the regimen in the experimental arm was designed because of our previous experience and data provided by Saltz et al. [16] on Grade 3 or 4 neutropenia and diarrhea in $53.8 \%$ and $22.7 \%$ of patients, respectively, who were treated with IFL for metastatic colon cancer. Therefore, we employed irinotecan at a relatively low dose of $80 \mathrm{mg} / \mathrm{m}^{2}$ with LV $200 \mathrm{mg} / \mathrm{m}^{2}$ and $5 \mathrm{FU} 450 \mathrm{mg} / \mathrm{m}^{2}$, weekly for four instead of six weeks, followed by the rest period. This dose of irinotecan was substantially reduced in comparison with the dose of $125 \mathrm{mg} /$ $\mathrm{m}^{2}$ which was utilized by Saltz et al. [20] in their adjuvant IFL regimen. On the other hand, treatment in Arm B was administered weekly for six consecutive weeks followed by the rest period that has resulted in combination with an increased by $10 \% 5 \mathrm{FU}$ dose in a statistically greater relative dose intensity for the fluoropyrimidine $(0.97 \mathrm{vs} .0 .86, P$ $<0.001)$. However, these differences are unlikely to have any impact on efficacy.

With the exception of leucopenia and neutropenia, which were higher in patients in Group A, there were no other significant differences in Grades 3 and 4 toxicities between the two regimens. The most frequently recorded Grade 3/4 toxicity was diarrhea in both treatment arms, followed by neutropenia. More specifically, severe diarrhea was observed in $15 \%$ and $12.5 \%$ of patients in Group A and Group B, respectively, while the corresponding rates for patients treated with either IFL or LV plus 5FU in the CALGB 89803 study were $31 \%$ and $35 \%$, respectively. On the other hand, Grade 3/ 4 neutropenia of the irinotecan plus LV plus 5FU arm was similar to that reported by Saltz et al. [20]. In this latter study, a higher incidence of treatment-related deaths has been reported with the IFL regimen compared to LV plus 5FU. However, in the present study this increased incidence was not observed in the experimental arm, possibly due to the dose level of irinotecan. Because of the higher incidence of severe neutropenia, more G-CSF was administered in patients who were allocated to irinotecan-based chemotherapy arm. 
Based on the results of the present and three previous negative trials [20-22], irinotecan should not be used in the adjuvant setting, because it adds no benefit when combined with either bolus or continuous-infusion 5FU. The question of why irinotecan has failed to demonstrate an advantage in the adjuvant setting while oxaliplatin is clearly of benefit still remains unanswered. Possible explanations involve clinical and pharmacologic aspects. Although the two head-to-head comparisons of infusional 5FU, LV, and oxaliplatin (FOLFOX) and infusional 5FU, LV, and irinotecan (FOLFIRI) in metastatic disease $[23,24]$ failed to show a significant difference in progression-free survival, each of these studies was remarkably underpowered to rule out a clinically meaningful difference between these regimens. Thus, the lack of adequately powered head-to-head comparisons between FOLFOX and FOLFIRI leaves open the possibility that FOLFOX may be superior to FOLFIRI [6].

In the PETACC-3 trial, the patients enrolled in the irinotecan arm experienced more dose reductions and more treatment discontinuation because of toxicity compared with the LV5FU2 arm. On the other hand, in this study after adjustment for imbalances in the TNM status between treatment groups, a multivariate analysis "rendered" a statistically significant DFS advantage in favor of the irinotecan $\operatorname{arm}(P=0.021)$, while a highly significant relapse-free survival advantage for the same arm could be achieved $(P=0.009)[21]$. Furthermore, the concept of individualized therapy based on prognostic and predictive molecular markers to better select patients who would benefit from a specific intervention is likely to be integral. Therefore, the possibility that irinotecan combined with LV and 5FU might be effective as adjuvant therapy in certain subsets of patients with colon cancer defined by various markers seems to be reasonable $[6,25]$. In this context, we have retrospectively analyzed by immunohistochemistry paraffin-embedded tumor tissues for detection of thymidylate synthase and topoisomerase I in patients treated with adjuvant chemotherapy within HeCOG protocols and we found that those expressing topoisomerase I seem to benefit from irinotecan-containing adjuvant chemotherapy [26]. Of particular interest is the CALGB 89803 study [20] that did not show, as previously mentioned, any differences in survival outcomes between IFL arm and LV plus 5FU arm. However, loss of tumor DNA mismatch repair (MMR) function could predict improved five-year DFS in patients treated with the IFL regimen as compared with those receiving LV plus 5FU [27]. Since the subset of patients who could derive a benefit from adjuvant irinotecan seems to represent a relatively small fraction, it is unlikely to be recognized when different populations are analyzed together. Finally, another speculation why irinotecan does not work in the adjuvant setting is the possibility that metastatic colon cancers may have different biological characteristics compared to primary tumors that could explain greater efficacy of irinotecan in metastatic disease.

\section{Conclusions}

The results of our trial demonstrated that weekly bolus irinotecan plus LV plus 5FU should not be used in the adjuvant setting for colon cancer. Since the number of agents that are potentially effective in the systemic treatment of completely resected stage II or III disease is increasing, it is important to ascertain which subgroups of patients will benefit from a specific treatment.

\section{Abbreviations}

ALT = alanine transaminase; $\mathrm{AST}=$ aspartate aminotransferase; $\mathrm{CA}=$ cancer antigen; CBC = complete blood cell; CEA = carcinoembryonic antigen; CNS = central nervous system; CPT-11 = irinotecan; $C T$ scan $=$ computed tomography; DFS = disease-free survival; ECG = electrocardiogram; FOLFIRI = 5fluorouracil, leucovorin and irinotecan; FOLFOX = 5fluorouracil, leucovorin and oxaliplatin; $5 F U=5$-fluorouracil; $H R=$ hazard rate; IFL regimen = irinotecan, 5fluorouracil and leucovorin; IV = intravenously; G-CSF = Granulocyte colony-stimulating factor; $L V=$ leucovorin; $M M R=$ mismatch repair; $\mathrm{OS}=$ overall survival; $\mathrm{WHO}=$ world health organization

\section{Acknowledgements}

Part of this work was presented at ESMO 35th, Milan 2010. The authors thank Ms Evita Fragou for monitoring the study and Ms Maria Moschoni from the HeCOG Data Office, for coordinating the data management

\section{Author details}

'Department of Clinical Therapeutics, "Alexandra" Hospital, University of Athens School of Medicine, Athens, Greece. ${ }^{2}$ Oncology Department, "Ippokration" Hospital, Athens, Greece. ${ }^{3}$ Department of Medical Oncology; "Papageorgiou" Hospital, Aristotle University of Thessaloniki School of Medicine, Thessaloniki, Greece. ${ }^{4}$ Department of Biostatistics, Hellenic Cooperative Oncology Group Data Office, Athens, Greece. ${ }^{5}$ Department of Medical Oncology, loannina University Hospital, loannina, Greece. ${ }^{6}$ Third Department of Medical Oncology, "Agii Anargiri" Hospital, Athens, Greece. 7 Laboratory of Histology and Embryology, Aristotle University of Thessaloniki School of Medicine, Thessaloniki, Greece. ${ }^{8} 1^{\text {st }}$ Propaedeutic Department of Surgery, "AHEPA" Hospital, Aristotle University of Thessaloniki School of Medicine, Thessaloniki, Greece. ${ }^{9} 2^{\text {nd }}$ Propaedeutic Department of Internal Medicine, Oncology Section, University General Hospital "Attikon", Athens, Greece. ${ }^{10} 2^{\text {nd }}$ Department of Medical Oncology, "Metropolitan" Hospital, Athens, Greece. ${ }^{11} 1^{\text {st }}$ Department of Medical Oncology, "Metropolitan" Hospital, Athens, Greece. ${ }^{12}$ Department of Pathology, "Ippokration" Hospital, Athens, Greece. ${ }^{13}$ Histopathology Department, "Alexandra" Hospital, Athens, Greece. ${ }^{14} 424$ Army General Hospital, Thessaloniki, Greece. ${ }^{15}$ Department of Pathology, "Papageorgiou" Hospital, Thessaloniki, Greece. ${ }^{16}$ Department of Medical Oncology, IKA Hospital, Thessaloniki, Greece. ${ }^{17}$ Division of Oncology, Department of Medicine, University Hospital of Patras, Rion, Greece.

\section{Authors' contributions}

CAP conceived the idea, participated in the design of the study, contributed to the acquisition of data and the statistical analysis, and drafted the manuscript. PP conceived the idea, participated in the design of the study and contributed to the acquisition of data. LM substantially contributed to the statistical analysis and critically revised the manuscript. MK, GP, AB, GB, NX, GK, CK, TE, IK, NP and FM were involved in the acquisition of data (treatment of the patients with surgery and chemotherapy). DM, GK, IP and IE centrally evaluated all histology specimens. MAD, DB, ES, GA, DP, TM and HPK made substantial contributions in the conception and design of the study and the acquisition of data and critically revised the manuscript. GF made substantial contributions in the conception and design of the study and the acquisition of data and critically revised the manuscript. All authors read and approved the final manuscript. 


\section{Competing interests}

The authors declare that they have no competing interests.

Received: 30 November 2010 Accepted: 31 January 2011 Published: 31 January 2011

\section{References}

1. Jemal A, Siegel R, Ward E, Murray T, Xu J, Thun MJ: Cancer statistics, 2007. CA Cancer J Clin 2007, 57:43-66.

2. Martenson JA Jr, Willett CG, Sargent DJ, Mailliard JA, Donohue JH, Gunderson LL, Charles R, Thomas CR Jr, Fisher B, Benson AB III, Myerson R, Goldberg RM: Phase III study of adjuvant chemotherapy and radiation therapy compared with chemotherapy alone in the surgical adjuvant treatment of colon cancer: results of intergroup protocol 0130. J Clin Oncol 2004, 22:3277-3283.

3. Chau I, Cunningham D: Adjuvant therapy in colon cancer - what, when and how? Annals of Oncology 2006, 17:1347-1359.

4. André T, Afchain P, Barrier A, Blanchard P, Larsen AK, Tournigand C, Louvet C, de Gramont A: Current Status of Adjuvant Therapy for Colon Cancer. Gastrointest Cancer Res 2007, 1:90-97.

5. Wolpin BM, Mayer RJ: Systemic treatment of colorectal cancer. Gastroenterology 2008, 134:1296-1310.

6. O'Connell MJ: Oxaliplatin or irinotecan as adjuvant therapy for colon cancer: the results are in. J Clin Oncol 2009, 27:3082-3084.

7. Garcia-Carbonero R, Supko JG: Current perspectives on the clinical experience, pharmacology, and continued development of the camptothecins. Clin Cancer Res 2002, 8:641-61.

8. Pizzolato JF, Saltz LB: The camptothecins. Lancet 2003, 361:2235-2242.

9. Shimada Y, Yoshino M, Wakui A, Nakao I, Futatsuki K, Sakata Y, Kambe M, Taguchi T, Ogawa N: Phase II study of CPT-11, a new camptothecin derivative, in metastatic colorectal cancer. J Clin Oncol 1993, 11:909-913.

10. Conti JA, Kemeny NE, Saltz LB, Huang Y, Tong WP, Chou TC, Sun M, Pulliam S, Gonzalez C: Irinotecan is an active agent in untreated patients with metastatic colorectal cancer. J Clin Oncol 1996, 14:709-715.

11. Rougier P, Bugat R, Douillard JY, Culine S, Suc E, Brunet P, Becouarn $Y$, Ychou Y, Marty M, Extra JM, Bonneterre J, Adenis A, Seitz JF, Ganem G, Namer M, Conroy T, Negrier S, Merrouche Y, Burki F, Mousseau M, Herait P, Mahjoubi M: Phase II study of irinotecan in the treatment of advanced colorectal cancer in chemotherapy-naive patients and patients pretreated with fluorouracil-based chemotherapy. J Clin Oncol 1997, 15:251-260

12. Kalofonos HP, Skarlos D, Bafaloukos D, Papakostas P, Bamias A, Janinis J, Timotheadou E, Kouvatseas G, Stavropoulos M, Economopulos T, Fountzilas G: A phase II study with CPT-11 plus leucovorin and bolus IV 5 -fluorouracil in patients with advanced colorectal carcinoma. Cancer Invest 2003, 21:855-862

13. Timotheadou E, Papakostas P, Tsavdaridis D, Basdanis G, Kalofonos H, Aravantinos G, Bafaloukos D, Fountzilas G: Irinotecan and oxaliplatin combination, as second-line treatment, in fluoropyrimidine-pretreated advanced colorectal cancer. A phase II study by the Hellenic Cooperative Oncology Group (HeCOG). Tumori 2005, 91:309-313.

14. Cunningham D, Pyrhönen S, James RD, Punt CJ, Hickish TF, Heikkila R, Johannesen TB, Starkhammar H, Topham CA, Awad L, Jacques C, Herait P: Randomised trial of irinotecan plus supportive care versus supportive care alone after fluorouracil failure for patients with metastatic colorectal cancer. Lancet 1998, 352:1413-1418.

15. Douillard JY, Cunningham D, Roth AD, Navarro M, James RD, Karasek P, Jandik P, Iveson T, Carmichael J, Alakl M, Gruia G, Awad L, Rougier P: Irinotecan combined with fluorouracil compared with fluorouracil alone as first-line treatment for metastatic colorectal cancer: A multicentre randomised trial. Lancet 2000, 355:1041-1047.

16. Saltz LB, Cox JV, Blanke C, Rosen LS, Fehrenbacher L, Moore MJ, Maroun JA, Ackland SP, Locker PK, Pirotta N, Elfring GL, Miller LL: Irinotecan plus fluorouracil and leucovorin for metastatic colorectal cancer: Irinotecan Study Group. N Engl J Med 2000, 343:905-914.

17. Kalofonos HP, Aravantinos G, Kosmidis P, Papakostas P, Economopoulos T, Dimopoulos M, Skarlos D, Bamias A, Pectasides D, Chalkidou S, Karina M, Koutras A, Samantas E, Bacoyiannis C, Samelis GF, Basdanis G, Kalfarentzos F, Fountzilas G: Irinotecan or oxaliplatin combined with leucovorin and 5fuorouracil as first-line treatment in advanced colorectal cancer: a multicenter, randomised, phase II study. Ann Oncol 2005, 16:869-877.
18. Fountzilas $G$, Zisiadis A, Dafni $U$, Konstantaras $C$, Hatzitheoharis $G$, Papavramidis S, Bousoulegas A, Basdanis G, Giannoulis E, Dokmetzioglou J, Katsohis C, Nenopoulou E, Karvounis N, Briassoulis E, Aravantinos G, Kosmidis $P$, Skarlos D, Pavlidis N: Fluorouracil and leucovorin with or without interferon alfa-2a as adjuvant treatment, in patients with highrisk colon cancer: a randomized phase III study conducted by the Hellenic Cooperative Oncology Group. Oncology 2000, 58:227-236.

19. André T, Boni C, Mounedji-Boudiaf L, Navarro M, Tabernero J, Hickish T, Topham C, Zaninelli M, Clingan P, Bridgewater J, Tabah-Fisch I, de Gramont A, Multicenter International Study of Oxaliplatin/5-Fluorouracil/ Leucovorin in the Adjuvant Treatment of Colon Cancer (MOSAIC) Investigators: Oxaliplatin, fluorouracil, and leucovorin as adjuvant treatment for colon cancer. N Engl J Med 2004, 350:2343-2351.

20. Saltz LB, Niedzwiecki D, Hollis D, Goldberg RM, Hantel A, Thomas JP, Fields AL, Mayer RJ: Irinotecan fluorouracil plus leucovorin is not superior to fluorouracil plus leucovorin alone as adjuvant treatment for stage III colon cancer: results of CALGB 89803. J Clin Oncol 2007, 25:3456-3461.

21. Van Cutsem E, Labianca R, Bodoky G, Barone C, Aranda E, Nordlinger B, Topham C, Tabernero J, André T, Sobrero AF, Mini E, Greil R, Di Costanzo F, Collette L, Cisar L, Zhang X, Khayat D, Bokemeyer C, Roth AD,

Cunningham D: Randomized phase III trial comparing biweekly infusional fluorouracil/leucovorin alone or with irinotecan in the adjuvant treatment of stage III colon cancer: PETACC-3. J Clin Oncol 2009, 27:3117-3125.

22. Ychou M, Raoul JL, Douillard JY, Gourgou-Bourgade S, Bugat R, Mineur L, Viret F, Becouarn Y, Bouché O, Gamelin E, Ducreux M, Conroy T, Seitz JF, Bedenne L, Kramar A: A phase III randomised trial of LV5FU2 + irinotecan versus LV5FU2 alone in adjuvant high-risk colon cancer (FNCLCC Accord02/FFCD9802). Ann Oncol 2009, 20:674-680.

23. Colucci G, Gebbia V, Paoletti G, Giuliani F, Caruso M, Gebbia N, Cartenì G, Agostara B, Pezzella G, Manzione L, Borsellino N, Misino A, Romito S, Durini E, Cordio S, Di Seri M, Lopez M, Maiello E, Montemurro S, Cramarossa A, Lorusso V, Di Bisceglie M, Chiarenza M, Valerio MR, Guida T, Leonardi V, Pisconti S, Rosati G, Carrozza F, Nettis G, Gruppo Oncologico Dell'ttalia Meridionale, et al: Phase III randomized trial of FOLFIRI versus FOLFOX4 in the treatment of advanced colorectal cancer: A multicenter study of the Gruppo Oncologico Dell' Italia Meridionale. J Clin Oncol 2005, 23:4866-4875.

24. Tournigand $C$, André T, Achille E, Lledo G, Flesh M, Mery-Mignard D, Quinaux E, Couteau C, Buyse M, Ganem G, Landi B, Colin P, Louvet C, de Gramont A: FOLFIRI followed by FOLFOX6 or the reverse sequence in advanced colorectal cancer: A randomized GERCOR study. $J$ Clin Oncol 2004, 22:229-237.

25. Tejpar S, Bertagnolli M, Bosman F, Lenz HJ, Garraway L, Waldman F, Warren R, Bild A, Collins-Brennan D, Hahn H, Harkin DP, Kennedy R, llyas M, Morreau H, Proutski V, Swanton C, Tomlinson I, Delorenzi M, Fiocca R, Van Cutsem E, Roth A: Prognostic and predictive biomarkers in resected colon cancer: current status and future perspectives for integrating genomics into biomarker discovery. Oncologist 2010, 15:390-404.

26. Kostopoulos I, Karavasilis V, Karina M, Bobos M, Xiros N, Pentheroudakis G, Kafiri G, Papakostas P, Vrettou E, Fountzilas G: Topoisomerase I but not thymidylate synthase is associated with improved outcome in patients with resected colorectal cancer treated with irinotecan containing adjuvant chemotherapy. BMC Cancer 2009, 9:339.

27. Bertagnolli MM, Niedzwiecki D, Compton CC, Hahn HP, Hall M, Damas B, Jewell SD, Mayer RJ, Goldberg RM, Saltz LB, Warren RS, Redston M: Microsatellite instability predicts improved response to adjuvant therapy with irinotecan, fluorouracil, and leucovorin in stage III colon cancer: Cancer and Leukemia Group B Protocol 89803. J Clin Oncol 2009, 27:1814-1821.

\section{Pre-publication history}

The pre-publication history for this paper can be accessed here: http://www.biomedcentral.com/1741-7015/9/10/prepub

doi:10.1186/1741-7015-9-10

Cite this article as: Papadimitriou et al:: A randomized phase III trial of adjuvant chemotherapy with irinotecan, leucovorin and fluorouracil versus leucovorin and fluorouracil for stage II and III colon cancer: A Hellenic Cooperative Oncology Group study. BMC Medicine 2011 9:10. 